\title{
Asymptotic Solution of Nonlinear Moment Equations for Constant-Rate Aerosol Reactors
}

\author{
B. D. SHAW
}

Mechanical and Aeronautical Engineering Department University of California Davis, CA 95616

(Received 8 August 1996)

\begin{abstract}
Nonlinear evolution equations based upon moments of the aerosol size distribution function are solved asymptotically for constant-rate aerosol reactors (i.e., where condensible monomer is added at a constant rate) operating in the free-molecular limit. The governing equations are nondimensionalized and a large parameter that controls nucleation behavior is identified. Asymptotic analyses are developed in terms of this parameter. Comparison of the asymptotic results with direct numerical integration of the governing equations is favorable. The asymptotic results provide a simplified analytical approach to estimating average particle sizes, particle number densities, and peak supersaturation values for constant-rate aerosol reactors.
\end{abstract}

Keywords: Asymptotic Expansions; Perturbations; Asymptotic Approximations; Heat And Mass Transfer; Phase Changes; Miscellaneous Topics

\section{INTRODUCTION}

The nucleation and growth of particles from a vapor is important in many practical systems, for example, with formation of particulates in the atmosphere or production of powders for industrial use. Some systems generate condensable material over a short time period (e.g., shock tubes). Others continuously transfer to or generate condensable material within a zone where appreciable nucleation occurs. These types of systems may be termed continuously-reinforced reactors, the simplest of which is the constant-rate aerosol reactor, as described by Friedlander [1]. With the constant-rate aerosol reactor, condensable material is generated at a constant rate within the reactor. This reactor is assumed to be spatially uniform in composition, and 
to have temporally-invariant temperatures and pressures (though the composition may change with time); smog chambers can approach conditions that are representative of constant-rate aerosol reactors.

Because of their practical and fundamental importance, constant-rate aerosol reactors have been the subject of a number of theoretical and computational studies. For example, Friedlander [1] has presented basic theory related to these reactors. Friedlander [2] has also developed a set of ordinary differential equations to model temporal variations of the moments of the aerosol distribution as well as the supersaturation of the condensible monomer.

In this paper, we consider the model advanced by Friedlander [2] for the dynamics of batch aerosol reactors operating in the free-molecular limit. This model provides nonlinear evolution equations for the first three moments of the aerosol size distribution, and includes the effects of nucleation and condensation; these evolution equations will be solved asymptotically in this paper. In this model, the Kelvin effect is assumed to be negligible, as is coagulation of particles. However, no restrictions are placed upon the aerosol size distribution (it is allowed to evolve as determined by the conservation equations), and the critical diameters $d_{p}{ }^{*}$ at which stable particles are nucleated are allowed to vary according to the Kelvin relationship, namely, $\mathrm{d}_{\mathrm{p}}{ }^{*}=4 \Psi \mathrm{V}_{1} /\left(\mathrm{k}_{\mathrm{b}} \mathrm{T} \ln S\right)$. The equations describing Friedlander's model are summarized below.

$$
\begin{gathered}
\frac{\mathrm{dN}}{\mathrm{dt}}=\mathrm{J} \\
\frac{\mathrm{dM}_{1}}{\mathrm{dt}}=\mathrm{Jd}_{\mathrm{p}}^{*}+\mathrm{B}_{1}(\mathrm{~S}-1) \mathrm{N} \\
\frac{\mathrm{dA}_{\mathrm{t}}}{\mathrm{dt}}=\mathrm{J}\left(\mathrm{g}^{*}\right)^{2 / 3} \mathrm{~s}_{1}+2 \pi \mathrm{B}_{1}(\mathrm{~S}-1) \mathrm{M}_{1} \\
\frac{\mathrm{dS}}{\mathrm{dt}}=\frac{\mathrm{Z}}{\mathrm{n}_{\mathrm{s}}}-\frac{\mathrm{Jg}^{*}}{\mathrm{n}_{\mathrm{s}}}-\frac{\mathrm{B}_{1} \mathrm{~A}_{\mathrm{t}}}{2 \mathrm{v}_{1} \mathrm{n}_{\mathrm{s}}}(\mathrm{S}-1)
\end{gathered}
$$

In these equations $S$ is supersaturation, $N$ is number density, $A_{t}$ is the total particle surface area per unit volume, $M_{1}$ is the first moment of the aerosol size distribution (essentially an average diameter), $\mathrm{t}$ is time, $\mathrm{Z}$ is the volumetric rate of generation of monomer molecules, $n_{s}$ the monomer number density at saturation conditions, $\mathrm{g}^{*}=\left[4 \pi /\left(3 \mathrm{v}_{1}\right)\right]\left[2 \Psi \mathrm{v}_{1} /\left(\mathrm{k}_{\mathrm{b}} \mathrm{T} \text { InS }\right)\right]^{3}$ is the number of monomers in a critical-size nucleus, $\Psi$ is surface tension, $\mathrm{T}$ is temperature, $\mathrm{k}_{\mathrm{b}}$ is Boltzmann's constant, $\mathrm{v}_{1}$ is the volume attributed to a monomer, $B_{1}=2 n_{s} v_{1}\left[k_{b} T /\left(2 \pi m_{1}\right)\right]^{1 / 2}\left(m_{1}\right.$ is the mass of a monomer mole- 
cule), $\mathrm{s}_{1}$ is the surface area attributed to a monomer in the liquid phase, and $\mathrm{J}$ is the particle nucleation rate, which is assumed to be described by classical nucleation theory such that $J=\left\{\left[2 \Psi / \pi m_{1}\right]^{1 / 2} n_{s}^{2} v_{1}\right\} S^{2} \exp \left(-E / n^{2} S\right)$ where $\mathrm{E}=16 \pi \Psi^{3} \mathrm{v}_{1}{ }^{2 /}\left(3 \mathrm{k}_{\mathrm{b}}{ }^{3} \mathrm{~T}^{3}\right)$. The parameter $\mathrm{E}$ is related to the activation energy for nucleation of a fresh particle. For typical situations, $\mathrm{E}$ is approximately $10^{2}$ or larger, such that nucleation rates are very sensitive to changes in S; this sensitivity will be exploited in the asymptotic analysis.

We will nondimensionalize Eqs. (1) - (4) by defining $\tau=t Z / n_{s}, n=$ $\mathrm{NZ} /\left(\mathrm{an}_{\mathrm{s}}\right), \mathrm{m}=\mathrm{M}_{1} \mathrm{Z} /\left(\mathrm{n}_{\mathrm{s}} \mathrm{ab}\right)$, and $\mathrm{a}_{\mathrm{t}}=\mathrm{A}_{\mathrm{t}} \mathrm{Z} /\left(\mathrm{n}_{\mathrm{s}} \mathrm{as}_{1} \mathrm{c}^{2 / 3}\right)$ where $\mathrm{a}=\left[2 \Psi / \pi \mathrm{m}_{1}\right]^{1 / 2}$ $\mathrm{n}_{\mathrm{s}}^{2} \mathrm{v}_{1}, \mathrm{~b}=4 \Psi \mathrm{v}_{1} /\left(\mathrm{k}_{\mathrm{b}} \mathrm{T}\right)$, and $\mathrm{c}=\left[4 \pi /\left(3 \mathrm{v}_{1}\right)\right]\left[2 \Psi \mathrm{v}_{1} /\left(\mathrm{k}_{\mathrm{b}} \mathrm{T}\right)\right]^{3}$. Inserting these variables into Eqs. (1) - (4) yields the dimensionless governing equations

$$
\begin{gathered}
\frac{\mathrm{dn}}{\mathrm{d} \tau}=\mathrm{S}^{2} \mathrm{e}^{-\mathrm{E} / \ln ^{2} \mathrm{~S}} \\
\frac{\mathrm{dm}}{\mathrm{d} \tau}=\frac{\mathrm{S}^{2}}{\ln \mathrm{S}} \mathrm{e}^{-\mathrm{E} / \ln ^{2} \mathrm{~S}}+\alpha(\mathrm{S}-1) \mathrm{n} \\
\frac{\mathrm{da} \mathrm{t}}{\mathrm{d} \tau}=\frac{\mathrm{S}^{2}}{\ln ^{2} \mathrm{~S}} \mathrm{e}^{-\mathrm{E} / \ln ^{2} \mathrm{~S}}+2 \alpha(\mathrm{S}-1) \mathrm{m} \\
\frac{\mathrm{d} \mathrm{S}}{\mathrm{d} \tau}=1-\gamma \frac{\mathrm{S}^{2}}{\ln ^{3} \mathrm{~S}} \mathrm{e}^{-\mathrm{E} / \ln ^{2} \mathrm{~S}}-\phi(\mathrm{S}-1) \mathrm{a}_{\mathrm{t}}
\end{gathered}
$$

where $\alpha=B_{1} n_{s}(b Z), \gamma=a c / Z$, and $\phi=B_{1} n_{s} a s_{1} c^{2 / 3} /\left(2 v_{1} Z^{2}\right)$. The initial conditions that will be used to develop solutions to Eqs. (5) - (8) are $\mathrm{n}(1)=\mathrm{m}(1)=\mathrm{a}_{\mathrm{t}}(1)=0, \mathrm{~S}(1)=1$, i.e., at the initial time $\tau=1$, the vapor is saturated and there are no particles present.

Equations (5) - (8) are nonlinear and coupled, which makes finding exact analytical solutions difficult. In fact, previous researchers who have investigated these equations have only determined solutions numerically. In this paper, however, asymptotic solutions to Eqs. (5) - (8) will be developed by exploiting the largeness of $\mathrm{E}$. These solutions will be compared with solutions obtained by direct numerical integration of Eqs. (5) - (8).

\section{COMPUTATIONAL RESULTS}

To begin, Eqs. (5) - (8) will first be integrated numerically to illustrate certain features of the solutions for the reader. The conditions under which Eqs. (5) - (8) were integrated correspond to the "model compound" considered 
by Warren and Seinfeld [5]. Property values for this compound as well as the assumed operating conditions for the aerosol reactor are summarized in Table (I). The values in Table (I) yield the result that the dimensionless surface-tension group $\Psi v_{1}{ }^{2 / 3} /\left(\mathrm{k}_{\mathrm{b}} \mathrm{T}\right)$ has a value of 1.85 for the chosen conditions. Based upon the arguments of Pratsinis et al. [3], it is therefore assumed that the Kelvin effect may be neglected in the calculations. In Table (I), the variable $\rho$ represents the density of monomer in the liquid phase, while $t_{\text {coll }}$ represents a characteristic time between collisions of monomer molecules at the saturation number density $n_{s}$. This value for $t_{\text {coll }}$ is calculated using the kinetic theory of gases. Classical nucleation theory is expected to break down when the characteristic time $n_{s} / Z$ for production of a monomer molecule is smaller than $t_{\text {coll }} / S$ (Warren and Seinfeld [5]). If we define the dimensionless monomer source rate $Z^{\prime}=t_{\text {coll }} Z / n_{s}$, we may roughly assume that classical nucleation theory begins to fail if $Z$ ' exceeds unity. In all of the calculations presented here, the variable $Z$ ' was never allowed to exceed unity. It is noted that by comparing their model with the simpler models of Friedlander [2] and Warren and Seinfeld [5], Rao and McMurry [4] suggest that if $2^{5 / 4} /\left(Z^{\prime}\right)^{1 / 2}>>1$, the Kelvin effect should not be dominant, and that even when $2^{5 / 4} /\left(Z^{\prime}\right)^{1 / 2}$ is of order unity errors associated with neglecting the Kelvin effect should not be overriding. The range $2.6<2^{5 / 4} /\left(Z^{\prime}\right)^{1 / 2}<260$ applied for the specific conditions examined here.

TABLE I Model Compound Properties

\begin{tabular}{lll}
\hline \multicolumn{1}{c}{ Property } & Value & \multicolumn{1}{c}{ Units } \\
\hline $\mathrm{T}$ & 298 & $\mathrm{~K}$ \\
$\mathrm{~W}_{1}$ (Mol. Wt.) & 100 & $\mathrm{~kg} / \mathrm{kmole}$ \\
$\Psi$ & 0.025 & $\mathrm{~N} / \mathrm{m}$ \\
$\mathrm{n}_{\mathrm{s}}$ & $2.43 \times 10^{14}$ & $\mathrm{~m}^{-3}$ \\
$\mathrm{t}_{\text {coll }}$ & 45 & $\mathrm{~s}$ \\
$\rho$ & 1000 & $\mathrm{~kg} / \mathrm{m}^{3}$ \\
\hline
\end{tabular}

Shown in Fig. (1) are values for the supersaturation $S$ plotted versus $\tau$. These results were obtained by numerically integrating Eqs. (5) - (8); all numerical integrations presented in this paper were generated using a fourth-order Runge-Kutta scheme. It is evident in this figure that an initial period exists where $S$ grows linearly with $\tau$. This period is terminated abruptly when the supersaturation peaks sharply and then begins to decrease. Figure (2) shows calculated histories for particle number densi- 


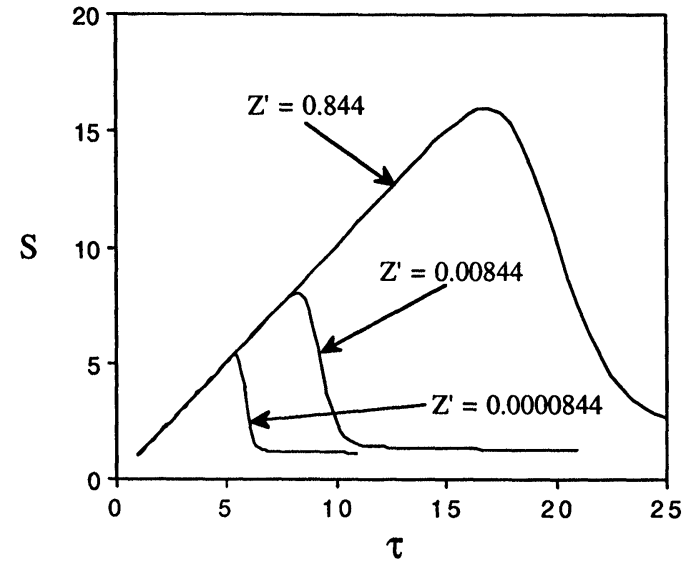

FIGURE 1 Plots of $S$ vs. $\tau$ obtained from integrating Eqs. (5) - (8)

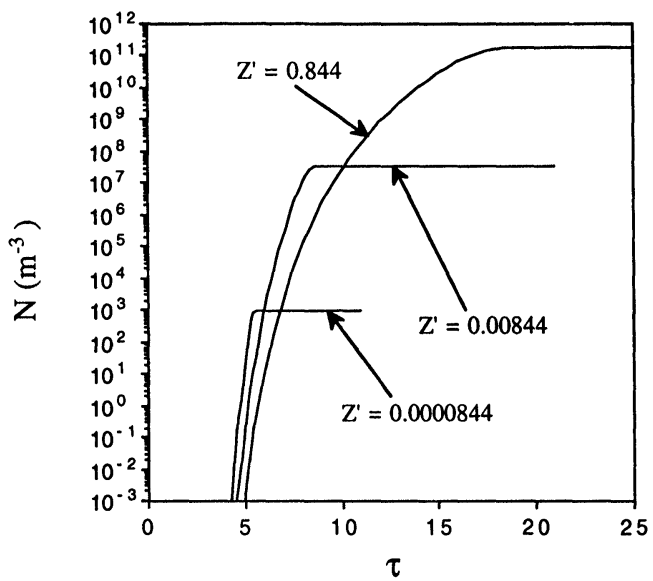

FIGURE 2 Plots of the number density $N$ vs. $\tau$ obtained from integrating Eqs. (5) - (8)

ties obtained by numerically integrating Eqs. (5) - (8). As would be anticipated, number densities grow very rapidly until $S$ peaks. After the supersaturation peaks, number densities then remain essentially constant because nucleation rates decrease in magnitude extremely rapidly when $S$ values drop even slightly below the peak $S$ value attained. Nucleation rates are largest when $S$ has peaked, and the nucleation of large numbers of particles over the time period when $\mathrm{S}$ is at its peak or slightly below is commonly termed a nucleation burst. 


\section{ASYMPTOTIC ANALYSIS}

The asymptotic analysis presented here will focus upon providing analytical expressions to predict peak $S$ values, particle number densities, and average particle sizes; these variables are frequently of interest for aerosols. The analysis will consider only the aerosol history prior to and including the nucleation burst. To begin, we may note that at $\tau=1$, the nucleation and condensation terms in Eqs. (5) - (8) are equal to zero, i.e., $d n / d \tau=d m / d \tau=$ $\mathrm{da}_{\mathrm{t}} / \mathrm{d} \tau=0$ while $\mathrm{dS} / \mathrm{d} \tau=1$. For $\tau$ (and hence $S$ ) larger than unity, the nucleation and condensation terms will be nonzero because $S$ will grow to values larger than unity. It is surmised, however, that $\tau$ will have to grow to values appreciably greater than unity before the nucleation and condensation terms become appreciable relative to unity and $\mathrm{dS} / \mathrm{d} \tau \approx 1$ is no longer a valid approximation; the applicability of this assumption is demonstrated by the previous computational results as well as by arguments presented below. If the nucleation and condensation terms in Eq. (8) (i.e., the last two terms on the right-hand-side of Eq. (8)) are negligible relative to unity, then Eq. (8) immediately yields the approximate solution

$$
\mathrm{S}=\tau+\ldots
$$

where "..." denotes small terms that are neglected. Equation (9) may be inserted into Eq. (5) to yield the differential equation

$$
\frac{\mathrm{dn}}{\mathrm{d} \tau}=\mathrm{f}(\tau) \exp \left(-\mathrm{E} / \ln ^{2} \tau\right)+\ldots
$$

where $f(\tau)=\tau^{2}$. The solution to Eq. (10) will provide an approximate expression to describe the transient particle number density for the time period where Eq. (9) is valid. To proceed, we will assume a solution of the form $n=g(\tau) \exp \left(-E / l^{2} \tau\right)$. Substituting this expression into Eq. (10) yields the ordinary differential equation for $g(\tau)$

$$
\frac{\mathrm{dg}}{\mathrm{d} \tau}+\frac{2 \mathrm{E}}{\tau \ln ^{3} \tau} \mathrm{g}=\mathrm{f}(\tau)+\ldots
$$

For $\mathrm{E} \gg>1$, the time derivative may be neglected in the first approximation, and an asymptotic solution to Eq. (11) is simply g = $f(\tau) \tau \ln ^{3} \tau /(2 E)+\ldots$. An asymptotic solution for the dimensionless particle number density for the time period where $S \approx \tau$ is then

$$
\mathrm{n}=\frac{\tau^{3} \ln ^{3} \tau}{2 \mathrm{E}} \exp \left(-\mathrm{E} / \ln ^{2} \tau\right)+\ldots
$$


It is noted that Eq. (12) may also be obtained by integrating Eq. (10), and then treating the resulting integral as a Laplace-type integral which can be asymptotically evaluated for $\mathrm{E} \rightarrow \infty$.

Equations (9) and (12) may now be inserted into Eq. (6) to yield

$$
\frac{\mathrm{dm}}{\mathrm{d} \tau}=\left[\frac{\tau^{2}}{\ln \tau}+\alpha(\tau-1) \frac{\tau^{3} \ln ^{3} \tau}{2 \mathrm{E}}\right] \exp \left(-\mathrm{E} / \ln ^{2} \tau\right)+\ldots
$$

An asymptotic solution to Eq. (13) may be developed by noting that this equation has the same general form as Eq. (10), that is, an algebraic function of $\tau$ multiplying the term $e^{-\mathrm{E} / \mathrm{ln}^{2} \tau}$. To develop a solution for $\mathrm{E} \gg 1$, we may follow the same general procedure as when Eq. (10) was solved. Upon doing this, it is found that Eq. (13) has the approximate solution

$$
\mathrm{m}=\frac{\tau \ln ^{3} \tau}{2 \mathrm{E}}\left[\frac{\tau^{2}}{\ln \tau}+\alpha(\tau-1) \frac{\tau^{3} \ln ^{3} \tau}{2 \mathrm{E}}\right] \exp \left(-\mathrm{E} / \ln ^{2} \tau\right)+\ldots
$$

We may also follow the same procedure to develop an approximate solution to Eq. (7), i.e., we can insert Eqs. (9) and (14) into Eq. (7). Doing this yields a differential equation that is the same general form as Eq. (10). The asymptotic solution of this equation eventually yields the approximate solution

$$
\mathrm{a}_{\mathrm{t}}=\frac{\tau \ln ^{3} \tau}{2 \mathrm{E}}\left[\frac{\tau^{2}}{\ln ^{2} \tau}+2 \alpha(\tau-1) \frac{\tau \ln ^{3} \tau}{2 \mathrm{E}}\left(\frac{\tau^{2}}{\ln \tau}+\alpha(\tau-1) \frac{\tau^{3} \ln ^{3} \tau}{2 \mathrm{E}}\right)\right] \exp \left(-\mathrm{E} / \ln ^{2} \tau\right)+\ldots(
$$

It is to be noted that Eqs. (12), (14) and (15) all contain the term exp($\left.E / n^{2} \tau\right)$, which dominates the behavior of $n, m$, and $a_{t}$ for $E>>1$, i.e., small changes in $\tau$ produce large changes in $\exp \left(-\mathrm{E} / \mathrm{ln}^{2} \tau\right.$ ) (so long as $\tau$ is not too large, which is always assumed here; typical values of $E$ are around 100 or greater, so $\tau$ may take on values that are quite large relative to unity before the term $\exp \left(-E / \ln ^{2} \tau\right)$ becomes insensitive to changes in $\left.\tau\right)$. Since $\exp \left(-E / \ln ^{2} \tau\right)$ appears in the asymptotic solution for $a_{t}, \tau$ must approach a critical time $\tau_{\mathrm{c}}$ very closely before the sum of the nucleation and condensation terms in Eq. (8) becomes appreciable relative to unity. We may specify a definite value for $\tau_{c}$ by requiring that it satisfy the equation

$$
\gamma \frac{\tau_{\mathrm{c}}^{2}}{\ln ^{3} \tau_{\mathrm{c}}} \exp \left(-\mathrm{E} / \ln ^{2} \tau_{\mathrm{c}}\right)+\phi\left(\tau_{\mathrm{c}}-1\right) \mathrm{a}_{\mathrm{t}, \mathrm{c}}=1
$$

where $a_{t, c}$ is the value of $a_{t}$ at the time $\tau=\tau_{c}$, and where Eq. (16) follows from setting $\mathrm{d} S / \mathrm{d} \tau=0$ in $\mathrm{Eq}$. (8). 
The approximation $\mathrm{S} \approx \tau$ is valid so long as $\tau$ does not approach $\tau_{\mathrm{c}}$ too closely. To analyze solution behaviors as $\tau$ approaches $\tau_{\mathrm{c}}$, we may define the rescaled time $\eta=2 \mathrm{E}\left(\tau-\tau_{\mathrm{c}}\right) /\left(\tau_{\mathrm{c}} \ln ^{3} \tau_{\mathrm{c}}\right)$ and the rescaled supersaturation $\sigma=2 \mathrm{E}\left(\mathrm{S}-\tau_{\mathrm{c}}\right) /\left(\tau_{\mathrm{c}} \ln ^{3} \tau_{\mathrm{c}}\right)$ as well as the rescaled variables $\Omega=\mathrm{n} / \mathrm{n}_{\mathrm{c}}$, $\Theta=m / m_{c}$ and $\Gamma=a_{t} / a_{t c}$ where $n_{c}, m_{c}$ and $a_{t c}$ are the values of $n, m$, and $a_{t}$ predicted by Eqs. (12), (14) and (15) at the time $\tau_{c} ; \Omega, \Theta$, and $\Gamma$ are all of order unity when $\eta$ is of order unity. The variable $\sigma$ is of order unity for $\tau$ within $O(1 / E)$ of $\tau_{c}$, and $\eta$ arises naturally when $d \sigma / d \eta$ is specified to be order unity or less for $\tau$ near $\tau_{\mathrm{c}}$. Inserting these new variables into Eqs. (5) - (8) and retaining only the largest terms when performing expansions for $\mathrm{E}>>1$ eventually yields the leading-order asymptotic expressions for the evolution expressions

$$
\begin{gathered}
\frac{\mathrm{d} \Omega}{\mathrm{d} \eta}=\mathrm{e}^{\sigma} \\
\frac{\mathrm{d} \Theta}{\mathrm{d} \eta}=\mathrm{x} \mathrm{e}^{\sigma}+(1-\mathrm{x}) \Omega \\
\frac{\mathrm{d} \Gamma}{\mathrm{d} \eta}=\mathrm{ye}^{\sigma}+(1-\mathrm{y}) \Theta \\
\frac{\mathrm{d} \sigma}{\mathrm{d} \eta}=1-\mathrm{ze}^{\sigma}-(1-\mathrm{z}) \Gamma
\end{gathered}
$$

where $\mathrm{x}=1 /\left(1+\mathrm{h}_{\mathrm{c}}\right), \mathrm{y}=1 /\left[1+2(1-\mathrm{x}) \mathrm{x}^{-2}\right], \mathrm{z}=1 /\left\{1+3 \mathrm{x}^{-1}(1-\mathrm{x})[1+2\right.$ $\left.\left.(1-\mathrm{x}) \mathrm{x}^{-2}\right]\right\}$, and $\mathrm{h}_{\mathrm{c}}=\alpha\left(\tau_{\mathrm{c}}-1\right) \tau_{\mathrm{c}} \mathrm{In}^{4} \tau_{\mathrm{c}} /(2 \mathrm{E})$. Values for $\mathrm{x}, \mathrm{y}$, and $\mathrm{z}$ will always be in the range $0-1$, and $x>y>z$. These variables represent the relative importances of nucleation and condensation. When these variables are close to unity, nucleation dominates over condensation in the conservation equations. When these variables are small relative to unity, which is typically the case in physical applications, the opposite situation holds. Equations (17) - (20) are to be solved subject to the matching conditions that as $\eta \rightarrow-\infty, \sigma=\eta, \Omega=\Theta=\Gamma=\mathrm{e}^{\eta}$. The exact solution $\sigma=-\ln \left(1+\mathrm{e}^{-}\right.$ $\eta), \Omega=\Theta=\Gamma=\ln \left(1+\mathrm{e}^{\eta}\right)$ is easily found when $\mathrm{x}=\mathrm{y}=\mathrm{z}=1$, that is, when $h_{c}=0$ and condensation effects are totally absent. An analytical solution for $h_{c}>0$ has not been found, so the equations are solved numerically for this case.

Shown in Figs. (3) - (5) are numerical solutions of Eqs. (17) - (20) for various values of $\mathrm{x}$. For $\mathrm{x}<1, \sigma$ peaks at a maximum value $\sigma_{\max }$, and $\Omega$, which is the rescaled number density, eventually approaches a constant (and maximum) value $\Omega_{\max }$; variations in $\Omega$ become small when 


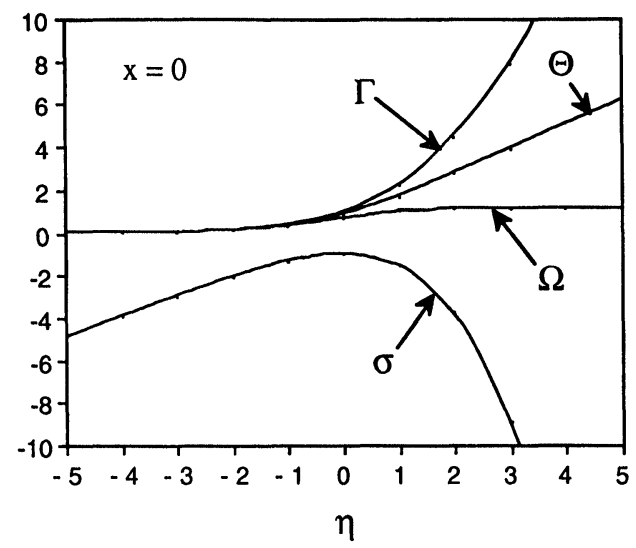

FIGURE 3 Plots of $\sigma, \Omega, \Theta$, and $\Gamma$ vs. $\eta$ for $\mathrm{x}=0$

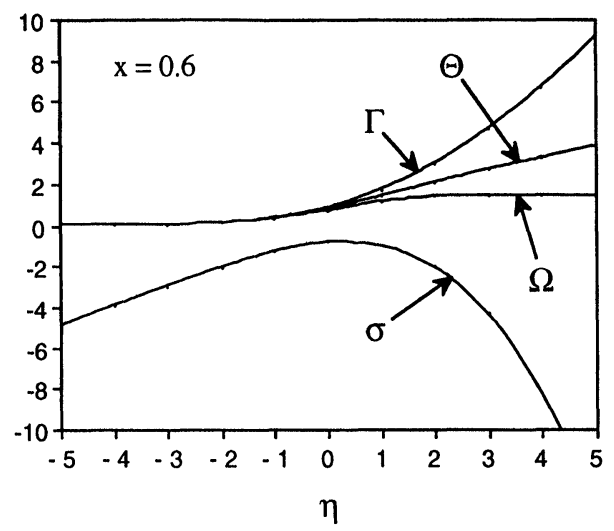

FIGURE 4 Plots of $\sigma, \Omega, \Theta$, and $\Gamma$ vs. $\eta$ for $\mathrm{x}=0.6$

$\sigma$ becomes less than -2 , and the nucleation burst is defined here to occur when $\sigma \geq-2$. Variations of $\sigma_{\max }$ and $\Omega_{\max }$ vs. $1-\mathrm{x}$ are shown in Fig. (6), where it is evident that $\sigma_{\max }$ and $\Omega_{\max }$ vary strongly as x approaches unity closely (note that the negative of $\sigma_{\max }$ is plotted). From the definitions of $\sigma$ and $\Omega$, we may develop the following equations to provide estimates for the maximum supersaturation $\left(\mathrm{S}_{\max }\right)$ and final number density $\left(\mathrm{n}_{\mathrm{tot}}\right)$

$$
\begin{gathered}
\mathrm{S}_{\max }=\tau_{\mathrm{c}}+\sigma_{\max } \frac{\tau_{\mathrm{c}} \ln ^{3} \tau_{\mathrm{c}}}{2 \mathrm{E}} \\
\mathrm{n}_{\text {tot }}=\Omega_{\max } \mathrm{n}_{\mathrm{c}} .
\end{gathered}
$$




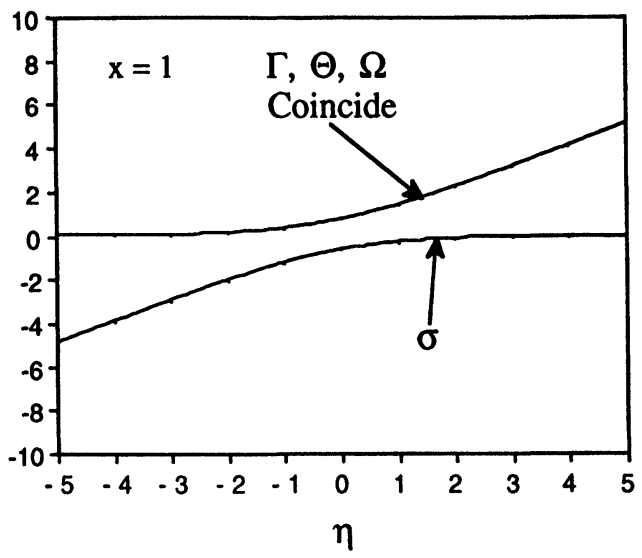

FIGURE 5 Plots of $\sigma, \Omega, \Theta$, and $\Gamma$ vs. $\eta$ for $\mathrm{x}=1$

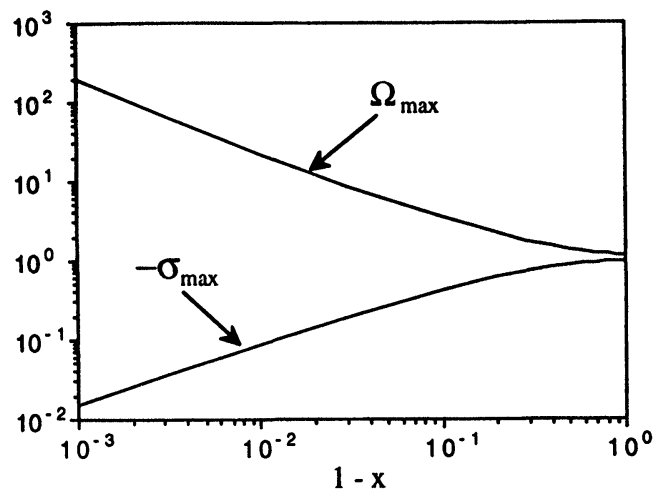

FIGURE 6 Plots of $-\sigma_{\max }$ and $\Omega_{\max }$ vs. $1-\mathrm{x}$

\section{COMPARISON OF COMPUTATIONAL AND ASYMPTOTIC RESULTS}

The asymptotic analyses have been applied to the same problem that was solved computationally (see Figs. (1) and (2)). In this section, comparisons between the asymptotic and computational results are made for the peak supersaturation $S_{\max }$ and the final number density $N_{\text {tot }}$. Figure (7) shows predictions for the peak supersaturation $S_{\max }$ attained as a function of $Z$ ' 


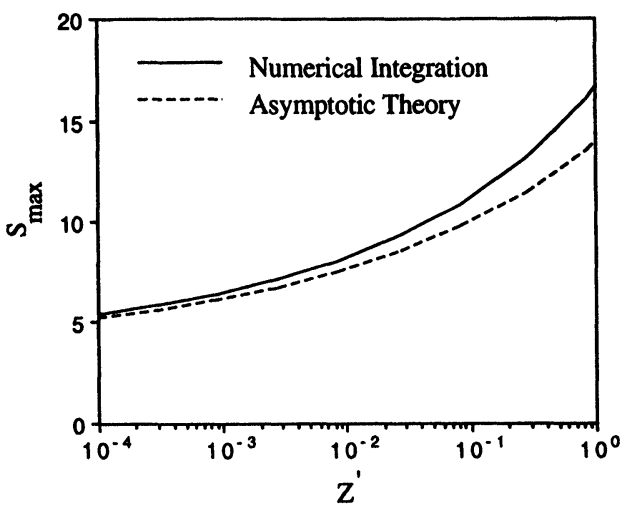

FIGURE 7 Comparison of predictions of the peak supersaturation $S_{\max }$ obtained from the asymptotic model and numerical integrations of Eqs. (5) - (8)

for the computational and asymptotic models (Eq. (21)). Qualitative and quantitative agreement between the numerical and asymptotic results is good, especially as Z' decreases in magnitude. Figure (8) shows plots of the final number density attained $\left(\mathrm{N}_{\text {tot }}\right)$ vs. A, where $\mathrm{N}_{\text {tot }}$ has units of particles per cubic meter. Agreement between the numerical and asymptotic results (Eq. (22)) is good, especially regarding qualitative trends over about eight orders of magnitude in predictions for number densities.

It is also of interest to evaluate average particle diameters during a nucleation burst. For this purpose, we will use area-averaged particle diameters $\left[A_{t} /(\pi N)\right]^{1 / 2}$. When use is made of the rescaled variables defined

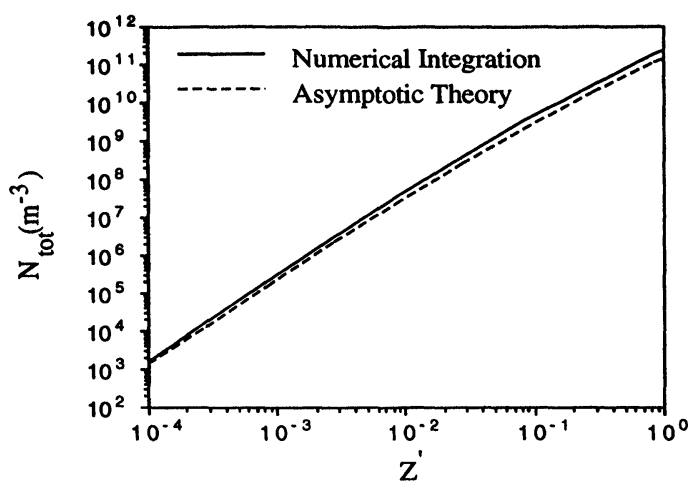

FIGURE 8 Comparison of predictions of the final number density $\mathrm{N}_{\text {tot }}$ obtained from the asymptotic model and numerical integrations of Eqs. (5) - (8) 
previously, theoretical area-averaged diameters, $d_{T H}=\left[A_{t} /(\pi N)\right]^{1 / 2}$, that are present during a nucleation burst can be expressed as

$$
\mathrm{d}_{\mathrm{TH}} \approx\left[\frac{\mathrm{s}_{1} \mathrm{c}^{2 / 3}}{\pi} \frac{1+2 \frac{1-\mathrm{x}}{\mathrm{x}^{2}}}{\ln ^{2} \tau_{\mathrm{c}}}\right]^{1 / 2}\left(\frac{\Gamma}{\Omega}\right)^{1 / 2} .
$$

Numerical integrations of Eqs. (17) - (20) show that prior to and during the time period where significant nucleation occurs, i.e., the nucleation burst (defined here as when $\sigma \geq-2$ ), $(\Gamma / \Omega)^{1 / 2}$ is always within the range $1-1.6$ for $0 \leq \mathrm{x} \leq 1$, indicating that average particle sizes do not change by large amounts during a nucleation burst. To provide estimates for average particle sizes during a nucleation burst, we will thus approximate $(\Gamma / \Omega)^{1 / 2}$ as having a value of unity, yielding Eq. (23) as an estimate for area-averaged particle diameters that exist during a nucleation burst.

$$
\mathrm{d}_{\mathrm{TH}} \approx\left[\frac{\mathrm{s}_{1} \mathrm{c}^{2 / 3}}{\pi} \frac{1+2 \frac{1-\mathrm{x}}{\mathrm{x}^{2}}}{\ln ^{2} \tau_{\mathrm{c}}}\right]^{1 / 2} .
$$

Table (II) provides results for predictions of area-averaged particle diameters obtained from the asymptotic model and the numerical calculations. The average diameters listed for the numerical calculations correspond to average diameters that existed when the peak supersaturation $S_{\max }$ was achieved. Also listed are theoretical average diameters defined by Eq. (23), as well as the $\tau_{\mathrm{c}}$ and $\mathrm{x}$ values that were calculated from the asymptotic theory. The results listed in Table (II) indicate that the asymptotic theory predicts average particle diameters quite well for the particular problem considered. It is noted that for these particular results the variable $\mathrm{x}$ happened to always be significantly smaller than unity; this may not always be the case in other situations.

TABLE II Comparison of area-averaged particle diameters from the asymptotic theory (Eq. (23)) and also from numerical integration. The subscripts "TH", and "N" refer to the asymptotic theory and numerical integrations of Eq. (5) - (8), respectively. Each diameter listed for the numerical integrations corresponds to the time when $S_{\max }$ was attained.

\begin{tabular}{cccrr}
\hline$Z^{\prime}$ & $\tau_{c}$ & $x$ & $d_{T H}(n m)$ & $d_{N}(n m)$ \\
\hline 0.844 & 14.79 & 0.244 & 8.45 & 7.75 \\
0.00844 & 7.77 & 0.0322 & 85.1 & 83.7 \\
0.0000844 & 5.24 & 0.00184 & 1870 & 1980 \\
\hline
\end{tabular}


The results listed in Table (II) were obtained assuming that particles were always within the free-molecular regime. For this to hold true, it is easily calculated that total aerosol reactor pressures must be quite low (a few Torr) for the largest particles considered; the smaller particles can withstand higher reactor pressures before the assumption of a free-molecular aerosol is violated. For coagulation to be negligible, the timescale $\Delta t$ for the onset and duration of the nucleation burst must be smaller than the characteristic time for coagulation $[1 /(\mathrm{N} \zeta)]$, where $\mathrm{N}$ is a characteristic aerosol number density and $\zeta$ is the collision frequency function. Estimates suggest that $\Delta \mathrm{t}<<[1 /(\mathrm{N} \zeta)]$ for all of the calculations performed here. In making these estimates, $\zeta$ values for collision rates between hard spheres in the free-molecular limit (Friedlander, [2]) were used, where the spheres were assumed to have the diameters and number densities in Table (II) and Fig. (8), respectively. Coagulation is expected to be negligible for the conditions under consideration here.

\section{CONCLUSIONS AND FUTURE DIRECTIONS}

An asymptotic model was developed to predict behaviors of constant-rate aerosol reactors operating with particles in the free-molecular regime. Results from the analyses provide simplified analytical criteria to predict relative importances of nucleation and condensation, and analytical expressions were presented to predict aerosol moments prior to and during a nucleation burst. The asymptotic model compared favorably with numerical integrations of the governing equations regarding peak supersaturations, average particle diameters, and particle number densities.

Finally, this analysis can be extended in several ways. For example, it is of interest to include the Kelvin effect and coagulation so that models could be developed that would apply to more general situations. It would also be worthwhile to extend the analysis to allow for variable monomer generation rates.

\section{References}

[1] S. K. Friedlander, The Behavior of Constant-Rate Aerosol Reactors, Aerosol Sci. Technol. 1 (1982), 3-13.

[2] S. K. Friedlander, Dynamics of Aerosol Formation by Chemical Reaction, Ann. N. Y. Acad. Sci. 404 (1983), 354-364. 
[3] S. E. Pratsinis, T. T. Kodas, M. P. Dudukovic, and S. K. Friedlander, Aerosol Reactor Design: Effect of Reactor Type and Process Parameters on Product Aerosol Characteristics, Ind. Eng. Chem. Process Des. Dev. 25 (1986), 634-642.

[4] R. P. Rao and P. H. McMurry, Nucleation and Growth of Aerosol in Chemically Reacting Systems: A Study of the Near-Collision-Controlled Regime, Aerosol Sci. Technol. 11 (1989), 120-132.

[5] D. R. Warren and J. H. Seinfeld, Nucleation and Growth of Aerosol from a Continuously-Reinforced Vapor, Aerosol Sci. Technol. 3 (1984), 135-53. 


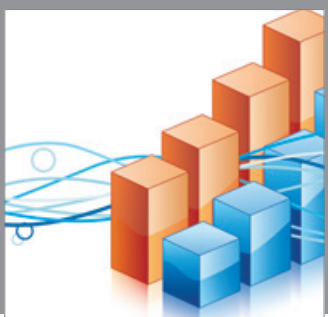

Advances in

Operations Research

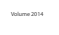

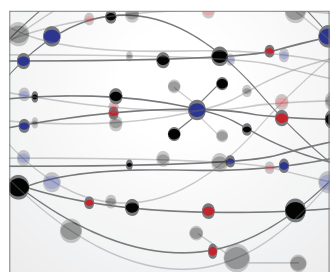

\section{The Scientific} World Journal
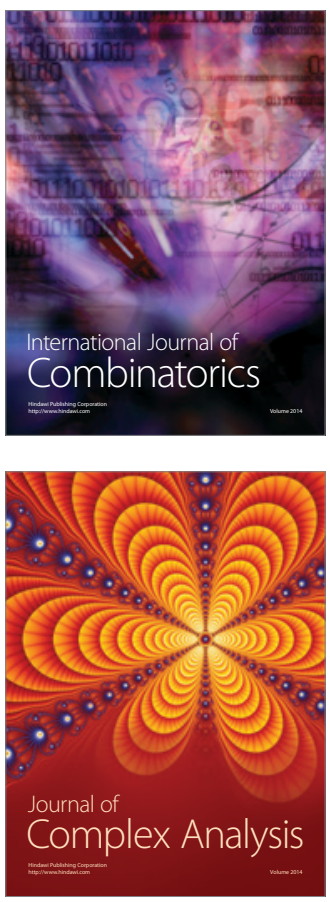

International Journal of

Mathematics and

Mathematical

Sciences
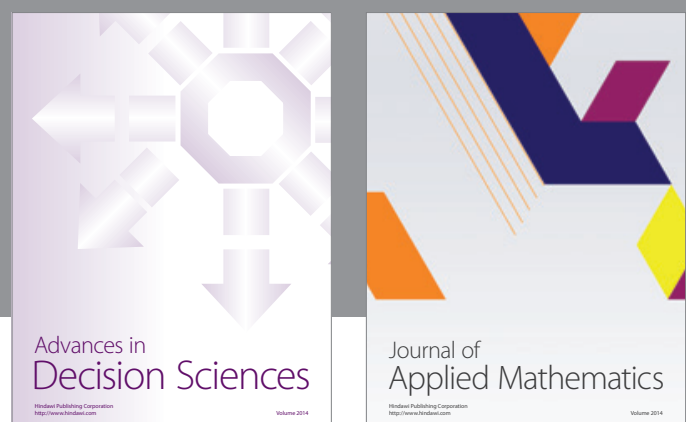

Journal of

Applied Mathematics
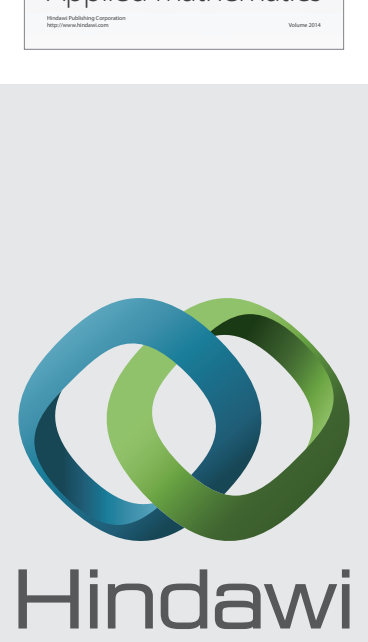

Submit your manuscripts at http://www.hindawi.com
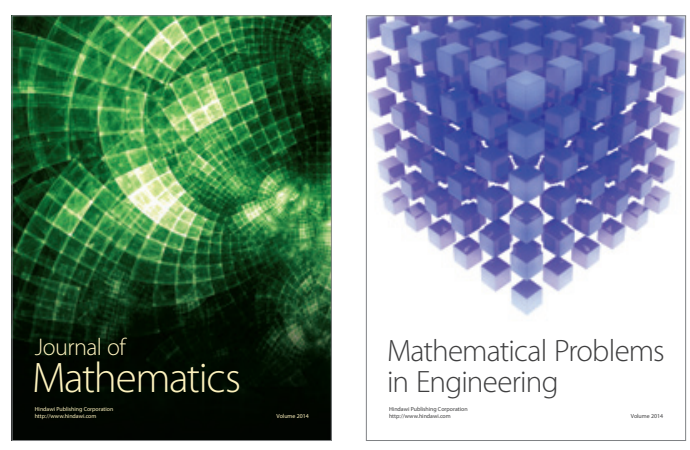

Mathematical Problems in Engineering
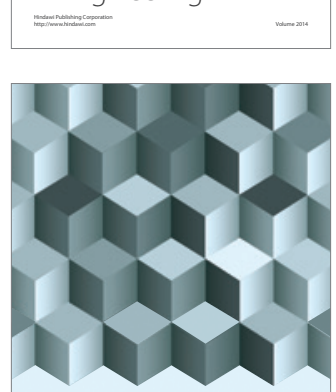

Journal of

Function Spaces
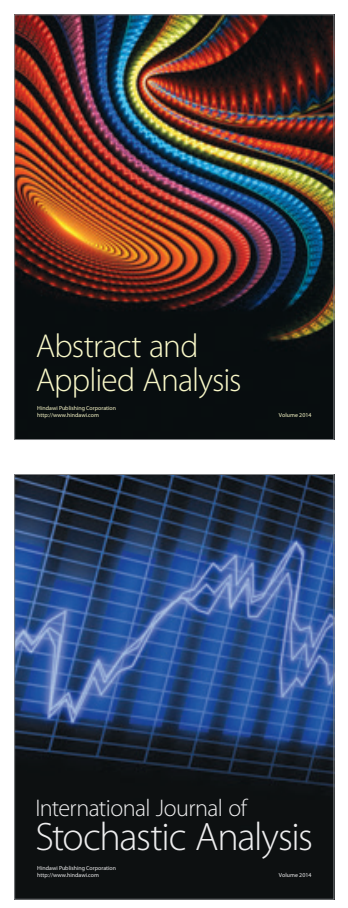

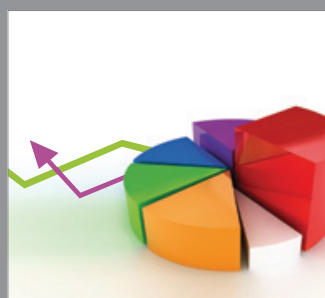

ournal of

Probability and Statistics

Promensencen
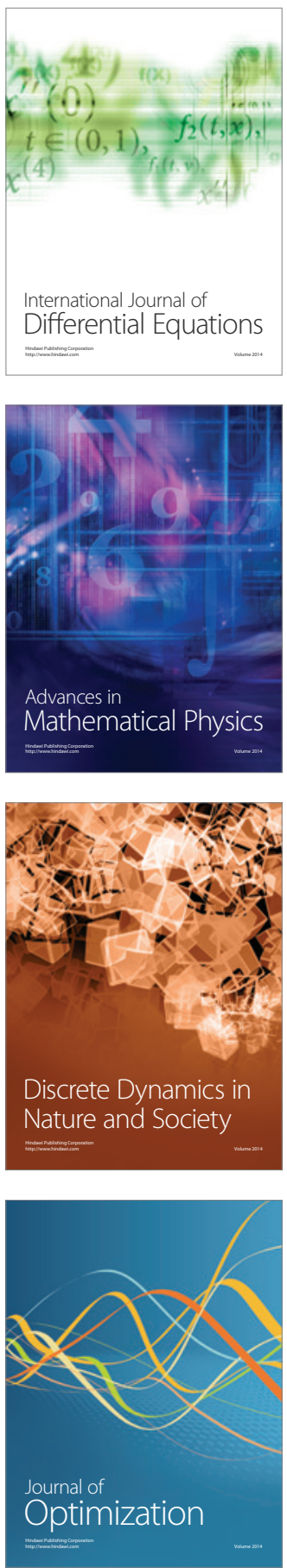